\title{
Long-lasting accretion-powered chemical heating of millisecond pulsars
}

\author{
E. M. Kantor ${ }^{\star}$, M. E. Gusakov†, \\ ${ }^{1}$ Ioffe Institute, Polytekhnicheskaya 26, 194021 St.-Petersburg, Russia
}

Accepted 2021 xxxx. Received 2021 xxxx; in original form 2021 xxxx

\begin{abstract}
We analyze the effect of magnetic field in superconducting neutron-star cores on the chemical heating of millisecond pulsars (MSPs). We argue that the magnetic field destroys proton superconductivity in some volume fraction of the stellar core, thus allowing for unsuppressed non-equilibrium reactions of particle mutual transformations there. The reactions transform the chemical energy, accumulated by a neutron star core during the low-mass X-ray binary stage, into heat. This heating may keep an NS warm at the MSP stage (with the surface temperature $\sim 10^{5} \mathrm{~K}$ ) for more than a billion of years after ceasing of accretion, without appealing to the rotochemical heating mechanism.
\end{abstract}

Key words: stars: neutron - stars: interiors - pulsars - nuclear reactions - ultraviolet: stars

\section{INTRODUCTION}

Rather high values of surface temperatures measured for two millisecond pulsars (MSPs), PSR J0437-4715 (Kargaltsev et al. 2004; Durant et al. 2012; Bogdanov et al. 2019) and PSR J2124-3358 (Rangelov et al. 2017), imply that some reheating mechanism should operate in these objects. A number of such mechanisms exists in the literature, the most promising are vortex creep (Alpar, Pines, Anderson, \& Shaham 1984), rotochemical heating in the crust (Gusakov, Kantor, \& Reisenegger 2015), and rotochemical heating in the core (Reisenegger 1995). Here we focus on the latter mechanism. Calculations by Petrovich \& Reisenegger (2010) and González-Jiménez, Petrovich, \& Reisenegger (2015) suggest that rotochemical heating can likely explain the observed temperatures of PSR J0437-4715 and PSR J21243358 if either the proton or neutron superfluid energy gaps (or both) are sufficiently large in the whole stellar core. However, the available analysis of the rotochemical heating did not account for the possible presence of the magnetic field in the core. Moreover, the effect of the neutron-star (NS) matter compression during the preceding accretion at the low-mass X-ray binary (LMXB) stage has never been studied. Our aim here is to fill these gaps.

The paper is organized as follows. Section 2 discusses available observations of MSP surface temperatures. In Section 3 we present general equations that govern the combined NS thermal and chemical evolution under compres-

\footnotetext{
* kantor@mail.ioffe.ru

† gusakov@astro.ioffe.ru
}

sion. The adopted physics input is described in Section 4. In Section 5 we analyze the effect of the core magnetic field on the chemical heating. Section 6 examines the effect of the accretion during the LMXB stage on the thermal states of MSPs. Section 7 contains discussion of the sensitivity of our results to various approximations made in the paper. We conclude in Section 8.

\section{OBSERVED SURFACE TEMPERATURES OF MILLISECOND PULSARS}

A millisecond pulsar with the best constraint on the surface temperature is PSR J0437-4715 (hereafter, 'J0437'; Kargaltsev et al. 2004; Durant et al. 2012). The mass of J0437 is measured to be $M=(1.3-1.58) M_{\odot}$ with $3 \sigma$ significance (Reardon et al. 2016). The rotation rate and spindown rate (accounting for the Shklovskii effect) equal, respectively, $\nu=173.7 \mathrm{~Hz}$ and $\dot{\nu}=-4.14 \times 10^{-16} \mathrm{~Hz} \mathrm{~s}^{-1}$. Effective redshifted (seen by a distant observer) surface temperature is estimated as $T_{\mathrm{s}}^{\infty}=(1.0-1.5) \times 10^{5} \mathrm{~K}$ (with about a $15 \%$ uncertainty dominated by the uncertainty in the extinction) for apparent, as seen by a distant observer, radius, $R_{\infty}=(18-13) \mathrm{km}$ (Durant et al. 2012). While Durant et al. (2012) obtained the surface temperature of J0437 by interpreting its thermal spectrum as blackbody emission, González-Caniulef et al. (2019) fitted the same spectrum with atmospheric models and found that the spectral fits favour a hydrogen atmosphere with the surface redshifted temperature $T_{\mathrm{s}}^{\infty}=(2.3 \pm 0.1) \times 10^{5} \mathrm{~K}$ and stellar coordinate radius $R=13.6_{-0.8}^{+0.9} \mathrm{~km}$. The lower/upper uncertainties 
provide $68 \%$ credible intervals. Later, by fitting new observational data from NICER with the hydrogen atmosphere model, Bogdanov et al. (2019) reported the local effective surface temperature $T_{\mathrm{s}}=\left(1.8_{-0.6}^{+0.2}\right) \times 10^{5} \mathrm{~K}$ and stellar coordinate radius $R=15.3_{-1.6}^{+2.0} \mathrm{~km}$ (90\% credible intervals).

Another pulsar with the measured surface temperature is PSR J2124-3358. The latter is estimated to be $(0.5-$ $2.1) \times 10^{5} \mathrm{~K}$ assuming the NS radius, as seen by a distant observer, $R_{\infty}=12 \mathrm{~km}$ (Rangelov et al. 2017). The rotation rate of PSR J2124-3358, $\nu=202.8 \mathrm{~Hz}$, and its spin-down energy loss rate, $\dot{E}=6.8 \times 10^{33} \mathrm{erg} \mathrm{s}^{-1}$, make this pulsar very similar to J0437.

There is also a set of upper limits on the surface temperatures of a number of millisecond pulsars (see, e.g., Schwenzer et al. 2017; Bhattacharya et al. 2017; Ho et al. 2019; Boztepe et al. 2019), which are, however, all rather high, being not very restrictive for rotochemical heating theory.

In view of the above mentioned facts, in what follows we only confront our results with the observations of the pulsar J0437, assuming that its effective redshifted surface temperature is $T_{\mathrm{s}}^{\infty}=(1.0-1.5) \times 10^{5} \mathrm{~K}$, in accordance with Durant et al. (2012); Bogdanov et al. (2019).

\section{GENERAL EQUATIONS AND OUR APPROACH}

To describe NS evolution under combined action of compression caused by accretion and spin-down, we follow the approach developed by Fernández \& Reisenegger (2005). Initially, this approach was applied to study departure from the beta-equilibrium of the spinning-down neutron star. However, an NS may accrete about $\sim 0.1 M_{\odot}$ during its evolution in LMXB (Özel et al. 2012; Antoniadis et al. 2016). Such a substantial amount of the accreted material compresses NS matter and could lead to strong deviations from chemical equilibrium. Thus, here we generalize the framework of Fernández \& Reisenegger (2005) to account, in addition, for the compression of matter due to accretion from the low-mass companion in an LMXB. Following Fernández \& Reisenegger (2005); Reisenegger et al. (2006), we assume that the redshifted chemical potentials $\mu_{\mathrm{n}}^{\infty}, \mu_{\mu}^{\infty}+\mu_{\mathrm{p}}^{\infty}$, and $\mu_{\mathrm{e}}^{\infty}+\mu_{\mathrm{p}}^{\infty}$ (as seen by a distant observer) are uniform because of the very efficient diffusion (Reisenegger 1997; Dommes et al. 2020), while the redshifted internal temperature, $T^{\infty}$, is uniform due to high thermal conductivity (Yakovlev et al. 2001). Here and below the subscripts b, n, p,e, $\mu$ refer to baryons, neutrons, protons, electrons, and muons, respectively. The temperature $T^{\infty}$ is driven by the thermal balance equation (Yakovlev et al. 1999; Fernández \& Reisenegger 2005)

$$
C \dot{T}^{\infty}=L_{\mathrm{acc}}^{\infty}-L_{\gamma}^{\infty}+\int_{V}\left(Q_{\mathrm{heat}}^{\infty}-Q_{\nu}^{\infty}\right) d V,
$$

where dot stands for the time derivative; $d V=4 \pi r^{2} \mathrm{e}^{\lambda / 2} d r$ is the proper volume element, with $\mathrm{e}^{\lambda}$ being the radial component of the metrics of a non-rotating reference star (the effect of rotation on the metrics is assumed to be small and is neglected in this equation). $C$ in equation (1) is the heat capacity of an NS and $Q_{\nu}^{\infty}$ is the neutrino emissivity. In what follows, we assume that the direct Urca (DUrca) processes
(Gamow \& Schoenberg 1941) are closed (their effect is discussed in Section 7) and account for the two main contributions to $Q_{\nu}^{\infty}$. The first one comes from the non-equilibrium modified Urca (MUrca) processes in the core:

$$
B_{i}+\mathrm{n} \rightarrow B_{i}+\mathrm{p}+\mathrm{l}+\bar{\nu}_{1}, \quad B_{i}+\mathrm{p}+\mathrm{l} \rightarrow B_{i}+\mathrm{n}+\nu_{1} .
$$

Here $B_{i}=\mathrm{n}$, p stands for, respectively, neutron and proton branches of MUrca reactions, $\mathrm{l}=\mathrm{e}, \mu$. The second contribution to $Q_{\nu}^{\infty}$ comes from the baryon-baryon bremsstrahlung:

$$
B_{i}+B_{k} \rightarrow B_{i}+B_{k}+\nu+\bar{\nu},
$$

where $B_{i}, B_{k}$ stands for $\mathrm{n}$ or $\mathrm{p} ; \nu, \bar{\nu}$ are neutrino and antineutrino of one of the three possible flavors. Generally, baryon-baryon bremsstrahlung is much weaker than MUrca processes. Still, if superfluidity (superconductivity) of neutrons (protons) strongly suppresses MUrca reactions, then bremsstrahlung with protons (neutrons) becomes the main contributor to $Q_{\nu}^{\infty}$. We emphasize, however, that the process (3) does not change the chemical composition of the stellar matter. Note that we do not account for the explicit contribution to $Q_{\nu}^{\infty}$ of the Cooper pairing neutrino emission process (e.g., Yakovlev et al. 2001), but discuss its possible role in Section 7. Further, $Q_{\text {heat }}^{\infty}$ in equation (1) represents the heat release in the non-equilibrium reactions

$$
Q_{\text {heat }}^{\infty}=\sum_{1=\mathrm{e}, \mu} \eta_{1}^{\infty} \Delta \Gamma_{1} \mathrm{e}^{\nu / 2},
$$

where $\eta_{1}^{\infty} \equiv \mu_{\mathrm{n}}^{\infty}-\mu_{1}^{\infty}-\mu_{\mathrm{p}}^{\infty}$ is the redshifted imbalance of chemical potentials (uniform throughout the core and vanishing in equilibrium); $\Delta \Gamma_{1}$ is the net production rate of particle species $l=\mathrm{e}, \mu$ due to reactions per unit volume (Yakovlev et al. 2001); $-\mathrm{e}^{\nu}$ is the time component of the metrics of a nonrotating reference star. Further,

$$
L_{\gamma}^{\infty}=4 \pi \sigma R^{2} T_{\mathrm{s}}^{4} \mathrm{e}^{\nu_{\mathrm{s}}}
$$

accounts for the photon emission from the surface. Here $R$ is the stellar coordinate radius, $\nu_{\mathrm{s}}=\nu(R)$. We relate the effective surface temperature in quiescence, $T_{\mathrm{s}}$, to the internal temperature, $T$, using the fitting formula (A8) from Potekhin et al. (1997), relevant to fully accreted envelope.

Finally, $L_{\mathrm{acc}}^{\infty}$ in equation (1) is the heating rate due to accretion

$$
L_{\mathrm{acc}}^{\infty} \approx \frac{\dot{M}}{m_{\mathrm{u}}} q \mathrm{e}^{\nu_{\mathrm{s}} / 2}
$$

where $m_{\mathrm{u}}$ is the nucleon mass unit, $\dot{M}$ is the average accretion rate (mass accreted per unit time of a distant observer), $q$ is the heat released per one accreted baryon because of non-equilibrium nuclear reactions in the crust (deep crustal heating). We approximate the redshift in the crust by the surface redshift, $\nu \approx \nu_{\mathrm{s}}$. The values of $\dot{M}$ and $q$ are rather uncertain. In our analysis we choose $\dot{M}=10^{-10} M_{\odot} /$ yr $\left(M_{\odot}\right.$ is the solar mass) and $q=0.5 \mathrm{MeV} /$ baryon, in accordance with Gusakov \& Chugunov (2020, 2021), who found that $q \lesssim 0.5 \mathrm{MeV} /$ baryon. Note, however, that our results are not sensitive to the choice of these values (see Section 6.2).

In addition to the thermal balance equation (1), one should also formulate evolution equations for the redshifted chemical potential imbalances, $\eta_{1}^{\infty}$. To establish these equations, let us introduce the vectors $\boldsymbol{\eta} \equiv\left(\delta \mu_{\mathrm{n}}, \eta_{\mathrm{e}}, \eta_{\mu}\right)$ (where $\delta \mu_{\mathrm{n}}$ is the departure of the neutron chemical potential from 
its equilibrium value; we introduce $\delta \mu_{\mathrm{n}}$ purely for mathematical reasons, in order to invert the matrix $\mathbf{A}_{(\mu)}$ appearing below) and $\boldsymbol{\delta} \boldsymbol{n} \equiv\left(\delta n_{\mathrm{b}}, \delta n_{\mathrm{e}}, \delta n_{\mu}\right)$. In the inner core, where muons are present, these vectors are related as

$$
\boldsymbol{\eta}=\mathbf{A}_{(\mu) j i} \boldsymbol{\delta} \boldsymbol{n},
$$

where the elements of the first row in the matrix $\mathbf{A}_{(\mu)}$ equal $\mathbf{A}_{(\mu) \mathrm{n} i}=\partial \delta \mu_{\mathrm{n}} / \partial n_{i}$, while the elements of the second and third rows equal $\mathbf{A}_{(\mu) l i}=\partial \eta_{1} / \partial n_{i}$; indices $l$ and $i$ run over $\mathrm{e}, \mu$ and $\mathrm{b}, \mathrm{e}, \mu$, respectively (note that, due to the quasineutrality condition, $n_{\mathrm{p}}=n_{\mathrm{e}}+n_{\mu}$, any thermodynamic quantity can be presented as a function of three number densities in the npe $\mu$ core). We multiply equation (7) by the matrix $\mathbf{A}_{(\mu) i j}^{-1}$, which is the inverse to $\mathbf{A}_{(\mu) j i}$, and integrate the result over the volume $V_{\mu}$, where muons are present:

$$
\left(\int_{V_{\mu}} \mathrm{e}^{-\nu / 2} \mathbf{A}_{(\mu) i j}^{-1} d V\right) \mathrm{e}^{\nu / 2} \boldsymbol{\eta}=\int_{V_{\mu}} \boldsymbol{\delta} \boldsymbol{n} d V .
$$

Here we factored out the uniform vector $\mathrm{e}^{\nu / 2} \boldsymbol{\eta}$. Then we follow the analogous procedure in the outer core, where muons are absent, and find:

$$
\left(\int_{V_{\mathrm{e}}} \mathrm{e}^{-\nu / 2} \mathbf{A}_{(\mathrm{e}) i j}^{-1} d V\right) \mathrm{e}^{\nu / 2} \boldsymbol{\eta}=\int_{V_{\mathrm{e}}} \boldsymbol{\delta} \boldsymbol{n} d V
$$

where $\mathbf{A}_{(\mathrm{e}) i j}^{-1}=0$ for the last row and the last column; integration goes over the outer core, where muons are absent. Then we sum up equations (8) and (9) to obtain

$$
\begin{array}{r}
\left(\int_{V_{\mu}} \mathrm{e}^{-\nu / 2} \mathbf{A}_{(\mu) i j}^{-1} d V+\int_{V_{\mathrm{e}}} \mathrm{e}^{-\nu / 2} \mathbf{A}_{(\mathrm{e}) i j}^{-1} d V\right) \mathrm{e}^{\nu / 2} \boldsymbol{\eta}= \\
=\left(\delta N_{\mathrm{b}}, \delta N_{\mathrm{e}}, \delta N_{\mu}\right),
\end{array}
$$

where $\delta N_{j}=N_{j}-N_{j}^{\text {eq }}$ is the difference between the actual particle number $N_{j}$ of species $j$ in the core and their equilibrium value, $N_{j}^{\text {eq }}$. In equilibrium both $\boldsymbol{\eta}$ and $\delta N_{j}$ must vanish. Multiplying equation (10) by the matrix $\mathbf{G}_{j i}$, which is the inverse to the matrix $\int_{V_{\mu}} \mathrm{e}^{-\nu / 2} \mathbf{A}_{(\mu) i j}^{-1} d V+\int_{V_{\mathrm{e}}} \mathrm{e}^{-\nu / 2} \mathbf{A}_{(\mathrm{e}) i j}^{-1} d V$, we find (see Fernández \& Reisenegger 2005):

$$
\eta_{1}^{\infty}=\mathbf{G}_{\mathrm{lb}} \delta N_{\mathrm{b}}+\mathbf{G}_{\mathrm{le}} \delta N_{\mathrm{e}}+\mathbf{G}_{\mathrm{l} \mu} \delta N_{\mu}
$$

where $\mathrm{l}=\mathrm{e}, \mu$. Taking time derivative, equation (11) can be presented as

$$
\dot{\eta}_{1}^{\infty}=\mathbf{G}_{\mathrm{lb}} \delta \dot{N}_{\mathrm{b}}+\mathbf{G}_{\mathrm{le}} \delta \dot{N}_{\mathrm{e}}+\mathbf{G}_{\mathrm{l} \mu} \delta \dot{N}_{\mu} .
$$

where $\delta \dot{N}_{i}=\dot{N}_{i}-\dot{N}_{i}^{\text {eq }}$. The equilibrium number of particle species $i$ in the core, $N_{i}^{\text {eq }}$, changes due to NS compression in the course of the accretion and/or NS spin-down. $\dot{N}_{i}^{\text {eq }}$ is responsible for building up the imbalances in the core. We determine $\dot{N}_{i}^{\text {eq }}$ following Hartle (1967); Hartle \& Thorne (1968); Fernández \& Reisenegger (2005) with all the necessary modifications caused by accretion. At the same time, an actual (non-equilibrium) number of particle species $i$ in the core, $N_{i}$, varies because of the two processes. The first process is the particle mutual transformations due to nonequilibrium MUrca reactions. These transformations tend to relax the imbalances $\eta_{1}$ generated by the accretion and/or spin-down of the star. The second one is the transformation of the bottom layers of the crust into the homogeneous matter of the core under compression. Since this transformation is a rather model-dependent process, in what follows we make a simplifying assumption that the number of baryons in the crust does not change with time. In other words, the accretion of $\delta A$ baryons increases the number of baryons in the core by the same value, $\delta A$. Obviously, $\delta N_{\mathrm{b}}=0$ in such a formulation of the problem. Moreover, we follow Gusakov \& Chugunov $(2020,2021)$ who suggested that the transformation of the crust matter into the core matter under compression is warranted by a specific instability that disintegrates nuclei in the inner layers of the crust into neutrons (with no admixture of protons and electrons). In such a paradigm, we have

$$
\dot{N}_{1}=\int_{\text {core }} \mathrm{e}^{\nu / 2} \Delta \Gamma_{1} d V .
$$

Note that, our conclusions are not really sensitive to the above assumptions about the physics near the crust-core boundary. We checked this by considering the two other limiting possible cases: (i) the accretion does not change the baryon number in the core; (ii) the accretion of $\delta A$ baryons increases the number of baryons in the core by $\delta A$, but the nuclei in the inner layers of the crust disintegrate to betaequilibrated homogeneous npe-matter of the core (not just neutrons). The cases (i) and (ii) lead to equivalent values of $\delta \dot{N}_{i}(i=\mathrm{b}, \mathrm{e}, \mu)$, and thus to a similar evolution of $\eta_{1}, T^{\infty}$, and $T_{\mathrm{s}}^{\infty}$ that only slightly differs from that presented below in this paper (see Figs. 6, 7, 8, and 9).

\section{PHYSICS INPUT}

To illustrate our results, we employ BSk24 EOS of the BSk (Brussels-Skyrme) family (Goriely et al. 2013; Potekhin et al. 2013; Pearson et al. 2018, 2019). This EOS allows for muons in the inner layers of an NS. In what follows, we consider $1.4 M_{\odot}$ NS model, for which DUrca processes are closed in the whole NS core (for BSk24 EOS DUrca processes operate in stars with $M>1.595 M_{\odot}$, where they accelerate the relaxation of the imbalances, see Section 7 ).

To calculate the neutrino emissivity, $Q_{\nu}$, due to baryonbaryon bremsstrahlung and non-equilibrium MUrca processes, as well as the net reaction rates, $\Delta \Gamma_{1}$, which appear in the evolution equations of Section 3, we follow the review article by Yakovlev et al. (2001), but account, in addition, for the enhancement of MUrca reaction rates reported by Shternin et al. (2018). More precisely, we use the formulas from Yakovlev et al. (2001) with the equilibrium emissivities for MUrca processes enhanced by a factor given by the equation (14) of Shternin et al. (2018). To calculate the heat capacity appearing in equation (1), we follow Yakovlev et al. (1999).

To simplify analysis, in the paper we assume that neutrons are nonsuperfluid, and discuss the effect of neutron superfluidity in Section 7, arguing that neutron superfluidity does not change the conclusions of the paper qualitatively, since neutron energy gap is expected to be noticeably lower than the proton gap.

We allow for the superconductivity of protons in the NS core and assume that, if present, proton superconductivity occupies the whole core. Moreover, in Section 5, when calculating the thermal states of an MSP with vanishing magnetic field, we, for simplicity, assume that the redshifted proton critical temperature is constant throughout the core and equals $T_{\mathrm{cp}}^{\infty}=2 \times 10^{9} \mathrm{~K}$. Note that microscopic calculations 


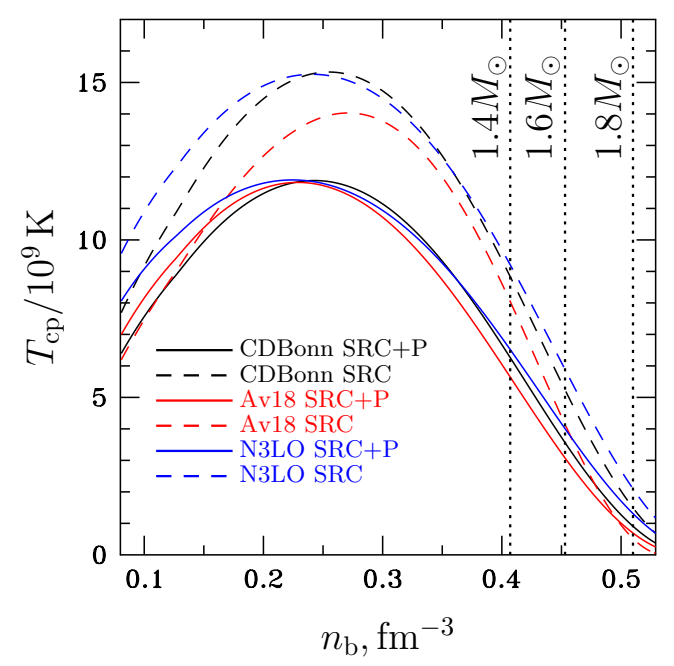

Figure 1. Local proton critical temperature $T_{\mathrm{cp}}$ in the NS core as a function of $n_{\mathrm{b}}$ for six models of Ding et al. (2016) (see their table I and notations there). Vertical dotted lines indicate central baryon number densities for NSs with $M=1.4 M_{\odot}, 1.6 M_{\odot}$, and $1.8 M_{\odot}$.

predict rather wide profiles of $T_{\mathrm{cp}}$, see, e.g., Fig. 1, where we demonstrate six critical temperature profiles from the paper by Ding et al. (2016), as functions of the baryon number density, $n_{\mathrm{b}} \cdot{ }^{1}$ Fig. 1 shows local (unredshifted) critical temperatures, which are independent of the stellar model. One can see that proton superconductivity extends over the whole core for NSs of moderate masses and $T_{\mathrm{cp}}$ is, generally, larger than $2 \times 10^{9} \mathrm{~K}$. Thus, $T_{\mathrm{cp}}^{\infty}=2 \times 10^{9} \mathrm{~K}$ can be considered as a lower limit on the real $T_{\mathrm{cp}}^{\infty}$ for a chosen model of an NS with $M=1.4 M_{\odot}$. Below we comment on how our results modify if real minimum of $T_{\mathrm{cp}}^{\infty}$ differs from $2 \times 10^{9} \mathrm{~K}$. Proton superconductivity with $T_{\mathrm{cp}} \gtrsim 2 \times 10^{9} \mathrm{~K}$ almost completely suppresses the proton heat capacity and protonbaryon bremsstrahlung processes (Yakovlev et al. 1999). Thus, we assume that, if protons are superconducting, they do not contribute to the heat capacity $C$; the contribution to $C$ from other particle species remains unaffected, while proton-baryon bremsstrahlung is completely suppressed. In turn, the reduction factors describing suppression of the nonequilibrium MUrca reactions by proton superconductivity are, generally, rather cumbersome and have been analyzed in detail by Villain \& Haensel (2005). Fortunately, in the limit $T \ll T_{\text {cp }}$ they can be substantially simplified. First, in this limit the nonequilibrium MUrca reactions only proceed if $\left|\eta_{1}\right|>\Delta_{\mathrm{p}}$ in the case of neutron branch of MUrca and if $\left|\eta_{1}\right|>3 \Delta_{\mathrm{p}}$ in the case of proton branch, where $\Delta_{\mathrm{p}}$ is the proton energy gap (Reisenegger 1997; Petrovich \& Reisenegger 2010). Petrovich \& Reisenegger (2010) proposed simple analytic expressions for the reduction factors of MUrca nonequilibrium reactions (see their formulas 20 and 21, and note that the corresponding expression B.15 in their appendix, which is equivalent to equations 20 and 21 , contains a typo). These expressions are valid for $\Delta_{\mathrm{p}} \gtrsim 30 k_{\mathrm{B}} T\left(k_{\mathrm{B}}\right.$ is the Stefan-Boltzmann constant). The above inequality is satisfied in the parameter range of our interest (e.g., for $T=10^{8} \mathrm{~K}$ and $T_{\mathrm{cp}}=2 \times 10^{9} \mathrm{~K}$ one has $\left.\Delta_{\mathrm{p}} \approx 35 k_{\mathrm{B}} T\right)$. We use these analytic expressions in our calculations.

When considering superconducting NS cores we, for the first time in this context, account for the magnetic field existing there. Depending on the density and microphysics model, protons in the NS core form a superconductor of either type I or type II (Glampedakis et al. 2011; Haskell \& Sedrakian 2018). ${ }^{2}$ If protons form a type II superconductor, the magnetic field is confined to the flux tubes (Abrikosov vortices) in the core; in the case of type I superconductor the field is contained in the macroscopic (but small) domains surrounded by the magnetic-field-free superconducting matter (de Gennes 1967). Protons are normal (nonsuperconducting) both inside the flux tubes (in the case of type-II superconductor) and inside the domains (in case of type-I superconductor) and we shall treat particle mutual transformations as fully unsuppressed there (see, e.g., Schaab et al. 1998, who used this approximation to describe MUrca reactions with localized proton excitations in the cores of flux tubes). We expect that this approximation gives correct order-of-magnitude estimates for the nonequilibrium MUrca reaction rates because the wavelengths of neutron, proton, and lepton quasiparticles in NS interiors are generally smaller than the typical sizes of the flux-tube cores and normal domains (e.g., Gusakov 2019; Dommes \& Gusakov 2017). Summarizing, we model the NS matter inside the flux tubes/normal domains as a uniform non-superconducting liquid. Note that, for simplicity, we neglect enhancement of the reaction rates by the magnetic field studied by Baiko \& Yakovlev (1999), because this enhancement is quite moderate for the magnetic field strengths reached inside the flux tubes/normal domains.

Clearly, the volume fraction occupied by the nonsuperconducting matter depends on the magnetic field strength in the core, $B$, and can be estimated as $\sim B / H_{\text {crit }}$, where $H_{\text {crit }}$ is the magnetic field value inside the flux tube/normal domain. In the case of type-II superconductor, $H_{\text {crit }}$ corresponds to the upper critical magnetic field, $H_{\mathrm{c} 2}$, while in type-I superconductor $H_{\text {crit }}=H_{\mathrm{c}}$ (de Gennes 1967). Both critical fields $H_{\mathrm{c} 2}$ and $H_{\mathrm{c}}$ vary throughout the core by a factor of few (Glampedakis et al. 2011; Lifshitz \& Pitaevskii 1980), see Fig. 2, and we adopt their typical value, $\sim 2 \times 10^{15} \mathrm{G}$, as $H_{\text {crit }}$ in our calculations. In what follows, both type-I and type-II superconductors are treated as described above, i.e., not discriminating between these two phases. Since the value of the magnetic field in the core is highly uncertain (see, e.g., Cruces, Reisenegger, \& Tauris 2019 and references therein) and can strongly deviate from the value of the NS dipole magnetic field, $B_{\text {dip }}$, we consider $B$ as a free parameter in the calculations below.

2 Other, more exotic phases are also possible, see an interesting recent work (Wood, Graber, \& Newton 2020) in this direction.
1 Actually, Ding et al. (2016) presented $T_{\mathrm{cp}}$ as a function of the proton Fermi momentum, $k_{\mathrm{Fp}}$. To obtain $T_{\mathrm{cp}}$ as a function of $n_{\mathrm{b}}$ we make use of the BSk24 EOS. 


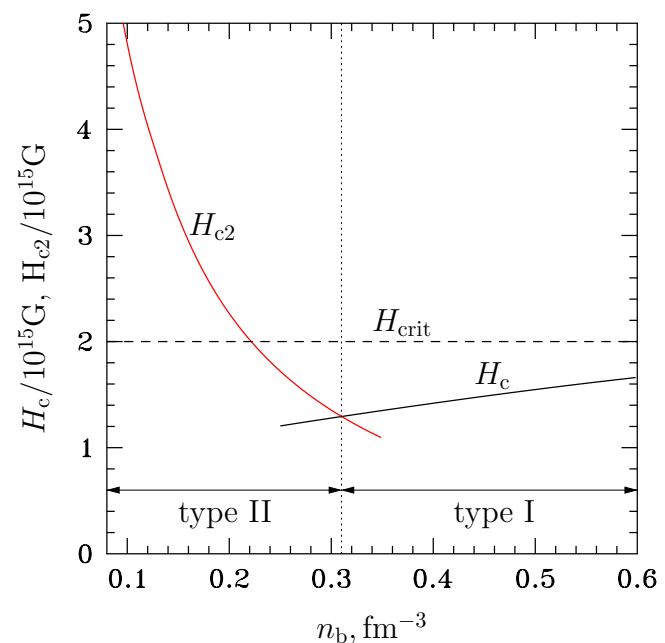

Figure 2. $H_{\mathrm{c}}$ and $H_{\mathrm{c} 2}$ in the NS core as functions of $n_{\mathrm{b}}$ for $T_{\mathrm{cp}}=$ $2 \times 10^{9} \mathrm{~K}$. Vertical dots show $n_{\mathrm{b}}$ corresponding to the interface between type I and type II superconductors. Dashes show adopted in the paper value of $H_{\text {crit }}$.

\section{THERMAL STATES OF SUPERCONDUCTING MAGNETIZED MSPS}

Here our aim is to analyze the role of the core magnetic field in the rotochemical heating of superconducting MSPs. But first, let us assume for a moment that the magnetic field is absent, protons are strongly superconducting in the whole NS core $\left(T^{\infty} \ll T_{\mathrm{cp}}^{\infty}\right), T_{\mathrm{cp}}$-profile is flat $\left(T_{\mathrm{cp}}^{\infty}=\right.$ const), while neutrons are normal. Since the chemical imbalances $\eta_{1}$ relax through the MUrca processes only, $\eta_{1}$ will grow until $\left|\eta_{1}^{\infty}\right|>\Delta_{\mathrm{p}}^{\infty}$, when the reactions of the MUrca neutron branch are open. After this condition is fulfilled for one of the imbalances, the reactions will prevent the subsequent growth of the corresponding $\eta_{1}^{\infty}$ by compensating the effect of compression (Reisenegger 1997). As a result, $\left|\eta_{1}^{\infty}\right|$ freezes at some equilibrium value slightly exceeding $\Delta_{\mathrm{p}}^{\infty}$ (see the dashed line in the last panel of Fig. 3 at $\log _{10} t>9.5$ ). Even small variation of $\eta_{l}^{\infty}$ results in a strong variation of the reaction rates tending to bring $\eta_{1}^{\infty}$ back to its equilibrium value. In what follows, we shall call such quasiequilibrium the 'steady state'. Higher values of $\Delta_{\mathrm{p}}^{\infty}$ correspond to higher values of $\left|\eta_{1}^{\infty}\right|$ in the steady state and higher energy release in the non-equilibrium reactions. Indeed, we can write: $\int_{V} Q_{\text {heat }}^{\infty} d V=\sum_{l} \eta_{1}^{\infty} \int_{V} \Delta \Gamma_{1} \mathrm{e}^{\nu / 2} d V=\sum_{1} \eta_{1}^{\infty} \dot{N}_{1}$ (see equations 4 and 13). To calculate $\dot{N}_{1}$ let us consider, for example, a situation when $\eta_{\mu}^{\infty}$ have reached the steady-state, while $\left|\eta_{\mathrm{e}}^{\infty}\right|<\Delta_{\mathrm{p}}^{\infty}$ (exactly this situation is realized in the fourth panel of Fig. 3). Then $\dot{N}_{e}=0$, since reactions with electrons are locked (we do not account for the lepton decay here, and discuss its role in Section 7), while the quasiequilibrium condition $\dot{\eta}_{\mu}^{\infty}=0$ prescribes $\dot{N}_{\mu}=\dot{N}_{\mu}^{\mathrm{eq}}+\mathbf{G}_{\mu \mathrm{e}} / \mathbf{G}_{\mu \mu} \dot{N}_{\mathrm{e}}^{\mathrm{eq}}$ (see equation 12). Thus, in the steady state $\dot{N}_{1}$ are driven by the compression rate and do not depend on the imbalances, while the heating rate appears to be proportional to the value of the imbalance in the steady state, or (approximately) to $\Delta_{\mathrm{p}}^{\infty}$. This property was used by Reisenegger (1997); Petrovich \& Reisenegger (2010); González-Jiménez et al. (2015) to explain high thermal luminosity of J0437.

Such an analysis, however, is valid only in the absence of the magnetic field. As we already discussed above, the magnetic field makes part of the stellar core with the volume fraction $\sim B / H_{\text {crit }}$ nonsuperconducting and allows for unsuppressed nonequilibrium reactions there. These reactions may efficiently relax the imbalances and prevent $\left|\eta_{1}^{\infty}\right|$ from growing to $\Delta_{\mathrm{p}}^{\infty}$.

To analyze the role of the magnetic field, we model the rotochemical heating of an MSP for different values of the core magnetic field, $B$. We assume that MUrca reactions are not suppressed inside the flux tubes (or normal domains), and are completely forbidden outside (Schaab et al. 1998). This is a good approximation, even for small $B$, as long as $\left|\eta_{1}^{\infty}\right|<\Delta_{\mathrm{p}}^{\infty}$ and $T^{\infty} \ll \Delta_{\mathrm{p}}^{\infty}$. For example, the reduction factor for the neutron branch of MUrca process is $\sim 2 \times 10^{-11}$ if we take $T^{\infty}=5 \times 10^{7} \mathrm{~K}, T_{\mathrm{cp}}^{\infty}=2 \times 10^{9} \mathrm{~K}$, and $\eta_{1}^{\infty}=0.8 \Delta_{\mathrm{p}}^{\infty}$. This value is much smaller than the nonsuperconducting volume fraction, $B / H_{\text {crit }}$, for the values of $B$ considered in the numerical examples below.

In this Section, we ignore the prehistory of the pulsar, namely, its compression at the LMXB stage. We assume that, initially (at the moment of time $t=0$ ), NS was in chemical equilibrium, and rotated at a spin frequency $\nu(t=$ $0)=\Omega(t=0) /(2 \pi)=300 \mathrm{~Hz}$; the NS temperature $T^{\infty}(t=$ $0)$ is assumed to be low. We also assume that the pulsar spins down due to magneto-dipole losses with the spin-down rate $\dot{\Omega}$ equal to

$$
\dot{\Omega}=-\frac{2 B_{\mathrm{dip}}^{2} R^{6} \Omega^{3}}{3 c^{3} I}
$$

where $I$ is the NS moment of inertia and $B_{\text {dip }}=1.6 \times 10^{8} \mathrm{G}^{3}$ While equation (14) describes the energy losses of a rotating dipole in vacuum, which is a rather crude emission model, its accuracy is quite sufficient for our analysis.

Figure 3 shows the evolution of $T^{\infty}, \eta_{\mathrm{e}}^{\infty}$, and $\eta_{\mu}^{\infty}$ for four values of the core magnetic field $B\left(B=10^{12}, 10^{9}, 10^{6}, 0 \mathrm{G}\right)$. In case of $B=0$ we assume $T_{\mathrm{cp}}^{\infty}=2 \times 10^{9} \mathrm{~K}$. One can see that, in the beginning, in all the panels chemical imbalances grow with the same rate. This rate is defined by the compression rate of the stellar matter; non-equilibrium reactions, which depend on the value of the imbalances, are still too slow to contribute to the evolution of the imbalances. Growth of the imbalances $\eta_{1}$ leads to the growth of the non-equilibrium reaction rates. As a result, at some moment non-equilibrium reactions come into play and start to compensate the effect of compression. The imbalances reach the steady state when this compensation becomes exact. After that, the imbalances stay in the steady state (see the arrow in the left panel). In the absence of the evolution of the compression rate $(\Omega \dot{\Omega}=$ const $)$, they would stay exactly constant. However, the compression rate decreases with time $(\Omega \dot{\Omega}$ decreases, see equation 14$)$ and the imbalances trace this decrease. Higher values of $B$ result in the lower steady-state imbalances and lower $T^{\infty}$. This is not surprising, since the higher $B$, the larger is the volume fraction, where nonequilibrium reactions are unsuppressed and can effectively relax $\eta_{1}^{\infty}$. Note that the above consideration with non-zero $B$ is valid only as long as $\eta_{1}^{\infty}<\Delta_{\mathrm{p}}^{\infty}$. Once $\eta_{1}^{\infty}$ reaches $\Delta_{\mathrm{p}}^{\infty}$, the reactions start to operate in the whole

3 Such value of $B_{\text {dip }}$ corresponds to the actual (i.e., accounting for the Shklovskii effect) spin-down rate of J0437. 

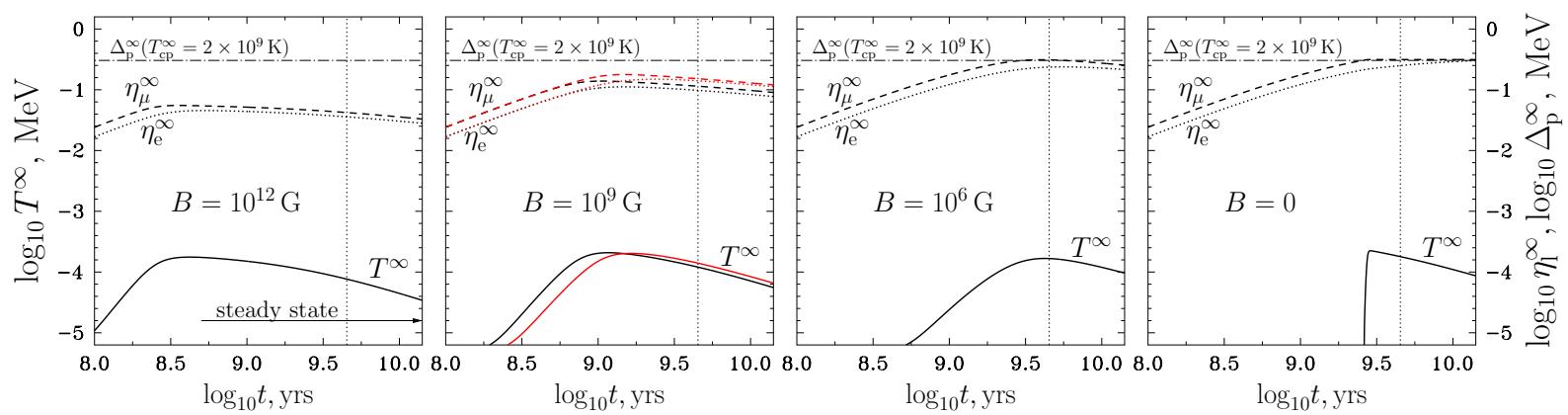

Figure 3. Internal stellar temperature $T^{\infty}$ (solid lines) and imbalances $\eta_{\mathrm{e}}^{\infty}$ and $\eta_{\mu}^{\infty}$ (dotted and dashed lines) versus time. Black lines account for the enhancement of MUrca reactions as discussed in Section 4, red lines (in the second panel from the left) disregard this effect. Vertical dots mark the time when $\nu \approx 173 \mathrm{~Hz}$ (the spin frequency of J0437). Horizontal dot-dashed lines show the redshifted proton superfluid gap, corresponding to $T_{\mathrm{cp}}^{\infty}=2 \times 10^{9} \mathrm{~K}$. At $t=0$ the star is cold and in chemical equilibrium; $\nu(t=0)=300 \mathrm{~Hz}$; $B_{\text {dip }}=1.6 \times 10^{8} \mathrm{G}$. We do not show the plot at $t<10^{8} \mathrm{yrs}$; it corresponds to the gradual growth of the imbalances.
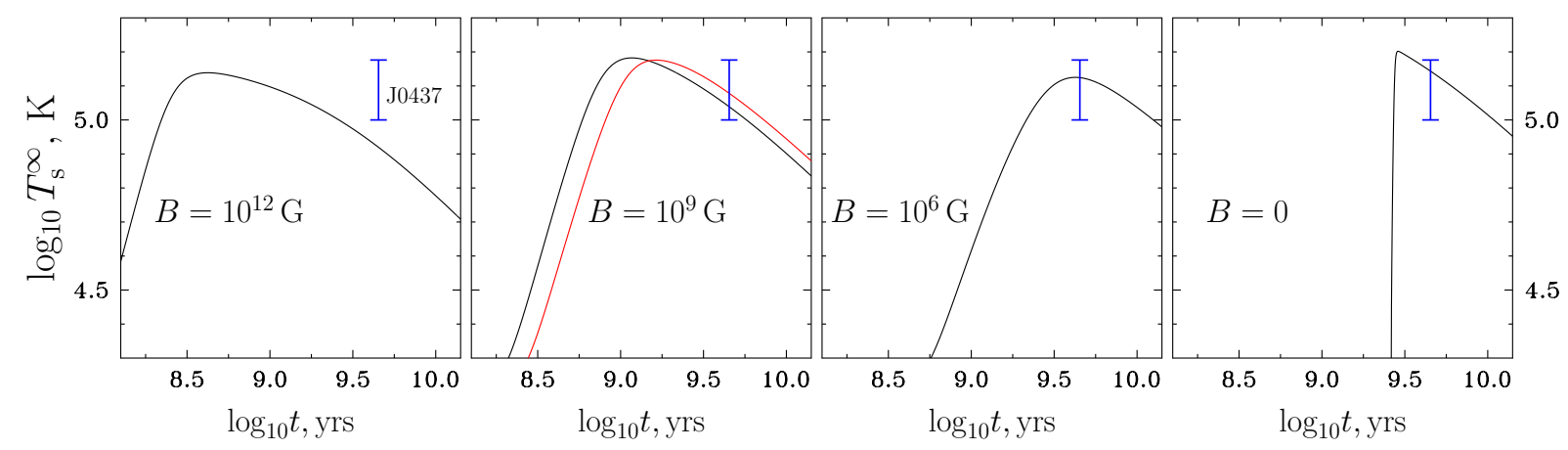

Figure 4. Surface stellar temperature $T_{\mathrm{s}}^{\infty}$ versus time for the same parameters as in Fig. 3. Notations are the same. Error bars show $T_{\mathrm{s}}^{\infty}$ for the pulsar J0437.

core and this stops further growth of $\eta_{1}^{\infty}$. For the reference we indicate $\Delta_{\mathrm{p}}^{\infty}$ for $T_{\mathrm{cp}}^{\infty}=2 \times 10^{9} \mathrm{~K}$ by dot-dashed line in Fig. 3. One sees that imbalances do not reach $\Delta_{\mathrm{p}}^{\infty}$ for $B \gtrsim 10^{6} \mathrm{G}$ and assumed spin-down parameters. In other words, the magnetic field $B \gtrsim 10^{6} \mathrm{G}$ reduces the efficiency of rotochemical mechanism. Figure 4 illustrates this point, explicitly showing the corresponding redshifted effective surface temperature, $T_{\mathrm{s}}^{\infty}$, for the same spin-down parameters and the same set of $B$ values as in Fig. 3. In Fig. 4 we also show the error bars for the measured redshifted effective surface temperature of J0437. We choose the horizontal coordinate corresponding to the actual spin rate of this MSP. The time coordinate is also in agreement with the estimated age of J0437, see Durant et al. (2012); Gonzalez \& Reisenegger (2010). One can see that at $B \gtrsim 10^{12} \mathrm{G}$ the rotochemical mechanism becomes unable to explain the observed temperature of J0437 for the adopted stellar model.

In the second panels of Figs. 3 and 4 we illustrate the effect of the enhancement of MUrca processes discussed in Shternin et al. (2018). For comparison, by red lines we show the results obtained neglecting the enhancement of MUrca reactions. One can see that, while the quantitative effect is obvious (red dashed and dotted lines pass higher in the steady state, because the reactions are not so effective), the picture does not change qualitatively, and thus our conclu- sions are not really sensitive to the accounting for the enhancement coefficients.

We should emphasize that, if the proton critical temperature profile does not extend over the whole NS core, then the unsuppressed reactions will proceed in the normal part of the core, which makes the role of the magnetic field in establishing the equilibrium negligible: Even at $B=0$ the imbalances will relax efficiently.

\section{EFFECT OF PRECEDING ACCRETION ON THE THERMAL STATES OF MSPS}

Let us now analyze how the prehistory of an MSP, namely the accretion at the LMXB stage, affects its subsequent thermal evolution.

We assume that the accretion proceeds with constant average rate $\dot{M}$ during some period of time $t_{\text {acc }}$ (in our numerical examples $\left.\dot{M}=10^{-10} M_{\odot} / \mathrm{yr}, t_{\mathrm{acc}}=10^{9} \mathrm{yrs}\right)$ and then smoothly (on a timescale $\sim 3 \times 10^{7}$ yrs, Tauris 2012) switches off. We also assume that during almost the whole LMXB phase (except for the beginning and final stages, see below for details), when accretion is active, an NS has an equilibrium frequency $\nu_{\text {eq }}$, defined by the condition that the accretion spin-up is balanced by the magneto-dipole spindown. In our numerical calculations we choose $\nu_{\mathrm{eq}}=300 \mathrm{~Hz}$. 


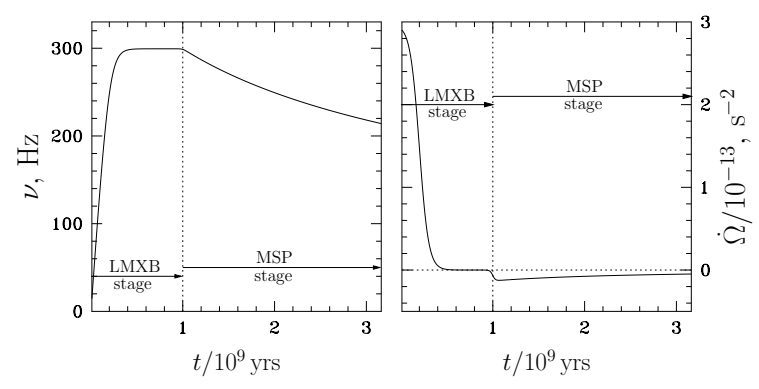

Figure 5. $\nu$ (left panel) and $\dot{\Omega}$ (right panel) versus time.

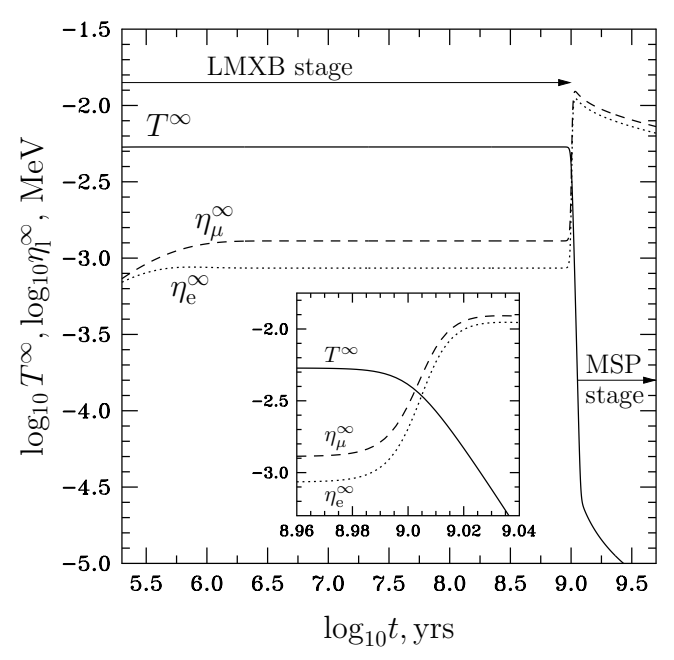

Figure 6. $T^{\infty}$ (solid line) and imbalances $\eta_{\mathrm{e}}^{\infty}$ (dotted line) and $\eta_{\mu}^{\infty}$ (dashed line) versus time. To highlight the accretion effect we neglect the effect of $\Omega$-variation on $T^{\infty}, \eta_{\mathrm{e}}^{\infty}$, and $\eta_{\mu}^{\infty}$. We do not show the plot at $\log _{10} t<5.3$, it corresponds to the gradual growth of the imbalances with time.

When the accretion smoothly switches off, the star starts to spin down. The spin-down rate increases gradually on the time-scale $\sim 3 \times 10^{7}$ yrs from zero to the value given by equation (14). In the beginning of the accretion phase we take into account an initial spin up of accreting NS up to the equilibrium frequency $\nu_{\text {eq. }}$. We choose the duration of this spin-up stage to be $\sim 2 \times 10^{8} \mathrm{yrs}$, in accordance with the observed $\dot{\nu}$ for some NSs in LMXBs during outbursts (see, e.g., Papitto et al. 2008; Patruno 2010; Papitto et al. 2011) and in accordance with the accretion torque modeling (Pringle \& Rees 1972; Ghosh \& Lamb 1979). Figure 5 shows the behavior of $\nu$ and $\dot{\Omega}$ with time. Then, using equations of Section 3, we model the joint thermal and chemical evolution of an NS.

\subsection{Nonsuperconducting NS matter}

First, we consider a normal (nonsuperconducting) NS. Figure 6 shows temporal evolution of $T^{\infty}, \eta_{\mathrm{e}}^{\infty}$, and $\eta_{\mu}^{\infty}$. In this figure we neglect $\Omega$ evolution to highlight the accretion effect (see Fig. 7 for the combined effect of accretion and $\Omega$ variation). The compression of NS material in the course of accretion drives an NS out of chemical equilibrium. As

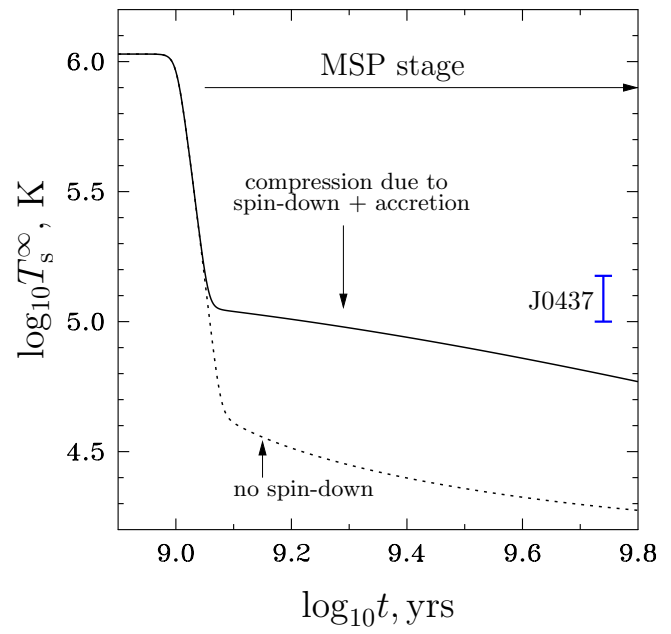

Figure 7. $T_{\mathrm{s}}^{\infty}$ normalized to $10^{5} \mathrm{~K}$ versus time. Dotted line is plotted assuming that there is no spin-down ( $\Omega$ is kept constant), solid line accounts for both compression due to accretion (at the LMXB stage) and NS spin-down. The evolution exclusively due to spin-down follows solid line very closely. Error bar shows $T_{\mathrm{s}}^{\infty}$ for the pulsar J0437.

a result, $\eta_{\mathrm{e}}^{\infty}$ and $\eta_{\mu}^{\infty}$ grow until they reach the equilibrium values when particle transformations become fast enough to compensate the subsequent growth of $\eta_{\mathrm{e}}^{\infty}$ and $\eta_{\mu}^{\infty}$. At the LMXB stage the value of $T^{\infty}$ corresponds to the equilibrium one, defined by the balance between the NS cooling and the deep crustal heating (as we already mentioned in Section 3, we assume $q=0.5 \mathrm{MeV}$ per accreted nucleon, see Gusakov \& Chugunov 2020, 2021). NS heating due to nonequilibrium particle transformations proceeding to restore the chemical equilibrium in the core also takes place, however, the energy released in these reactions (chemical heating) is much smaller than the deep crustal heating. When accretion ceases at $t \sim 10^{9} \mathrm{yrs}$, the NS internal temperature $T^{\infty}$ drops on a typical NS cooling timescale. However, at some value of $T^{\infty}$, NS cooling becomes approximately balanced by the chemical heating in the core. Then the temperature fall slows down and subsequently (at $t \gtrsim 10^{9.15} \mathrm{yrs}$ ) $T^{\infty}$ evolves on the timescale of chemical evolution. Generally, after the accretion ceases, the imbalances are driven by two trends. First, $\eta_{\mathrm{e}}^{\infty}$ and $\eta_{\mu}^{\infty}$ tend to relax to zero by means of particle transformations. Second, the NS matter continues to be compressed due to NS spin-down that maintains the non-zero imbalances. Note, however, that Fig. 6 neglects the spin-down ( $\Omega$ is artificially kept constant) so that in this figure the imbalances at the MSP stage are driven by the relaxation through non-equilibrium reactions only. The reader can notice a sharp increase of $\eta_{\mathrm{e}}^{\infty}$ and $\eta_{\mu}^{\infty}$ at $t \sim 10^{9} \mathrm{yrs}$, when accretion ceases (see also inset in Fig. 6). It is related to the fact that when the accretion rate starts to decrease (we remind that this process lasts $\sim 3 \times 10^{7} \mathrm{yrs}$ ), the NS temperature rapidly falls down. Driven by strong temperature dependence, the reaction rates decrease as well, making the relaxation of the imbalances inefficient. As a result, $\eta_{\mathrm{e}}^{\infty}$ and $\eta_{\mu}^{\infty}$ get an opportunity to grow up. However, at some moment the growth of the imbalances stops. The main reason for that is that then the accretion rate becomes too low to provide sufficient compression of NS matter. 
Fig. 7 shows the dependence $T_{\mathrm{s}}^{\infty}(t)$. The surface temperature traces $T^{\infty}$ behavior. Solid line accounts for the compression of NS material in the course of both accretion and spin-down ( $\Omega$ is now allowed to vary); dotted line shows the effect of accretion only $(\dot{\Omega}=0)$. If we account for NS spin-down only, neglecting compression of the star at the LMXB stage $\left(t \lesssim 10^{9} \mathrm{yrs}\right)$ by the accreted material, we obtain the line that practically coincides with the solid line. Figure 7 implies that in the normal NSs compression due to accretion alone (dots) does not generate sufficient imbalances to maintain relatively high NS surface temperatures after the accretion ceases, comparable to those observed in some MSPs. The reason for that is reactions of particle transformations are fast enough to keep the NS core close to the equilibrium $\left(\eta_{\mathrm{e}}^{\infty} \approx \eta_{\mu}^{\infty} \approx 0\right)$ despite the strong compression caused by accretion. Variation of $q, \dot{M}$, as well as account for $\dot{M}$ temporal dependence do not change the situation qualitatively.

\subsection{Superfluid/superconducting NS matter}

Baryon superfluidity/superconductivity suppresses the nonequilibrium reaction rates. Thus, in superfluid/superconducting NSs, we can expect that higher values of the imbalances will be reached at the LMXB stage, which can affect the NS surface temperature at the subsequent MSP stage. To illustrate this point, let us assume that protons are strongly superconducting, while neutrons are normal. We allow for non-zero magnetic field in the core (characterized by the parameter $B$ ) and model the NS evolution assuming that the proton energy gap is high enough, so that the inequality $\eta_{1}^{\infty}<\Delta_{\mathrm{p}}^{\infty}$ is always satisfied, and MUrca reactions can be treated as fully suppressed outside the flux tubes/normal domains. Figure 8 shows $T_{\mathrm{s}}^{\infty}$ for the three values of $B$. Lower magnetic fields result in higher $T_{\mathrm{s}}^{\infty}$ after cessation of accretion (at $t>10^{9} \mathrm{yrs}$ ). This is not surprising, since the imbalances generated in the star during its evolution are higher for lower $B$, see Fig. $9 .{ }^{4}$ At the same time, for sufficiently large magnetic fields such as $B=10^{12} \mathrm{G}$ the generated imbalances are too low to explain the surface temperature of J0437. Another interesting feature is that, for MSPs with even vanishing spin-down rate, $T_{\mathrm{s}}^{\infty}$ can be relatively high during some period of time after accretion switches off and NS becomes an MSP. For example, for $B=10^{9} \mathrm{G}$ the surface temperature remains $T_{\mathrm{s}}^{\infty} \gtrsim 10^{5} \mathrm{~K}$ during approximately $10^{9} \mathrm{yrs}$ after accretion ceases at vanishing spin-down rate (see dotted line in the middle panel of Fig. 8). In turn, for $B=10^{6} \mathrm{G}$ this period lasts approximately $4.5 \times 10^{9} \mathrm{yrs}$. During this time $T_{\mathrm{s}}^{\infty}$ is supported by relaxation of the imbalances generated during compression of matter by accretion at the LMXB stage. In the case of $B=10^{6} \mathrm{G}$ this heating could support the observed temperature of J0437 even in the absence of the pulsar spin-down.

Thus, our results imply that temperature of an MSP

4 Note that, in the case of $B=10^{6} \mathrm{G}$ (third panel in Figs. 8 and 9) the condition $\eta_{1}^{\infty}<\Delta_{\mathrm{p}}^{\infty}$ assumed in our calculations is only fulfilled for $T_{\mathrm{cp}}$-profiles with $T_{\mathrm{cp}}^{\infty} \gtrsim 5 \times 10^{9} \mathrm{~K}$, while in the cases $B=10^{9} \mathrm{G}$ and $B=10^{12} \mathrm{G}$ the condition $T_{\mathrm{cp}}^{\infty} \gtrsim 2 \times 10^{9} \mathrm{~K}$ is sufficient. may be directly related to the evolution of the star at the LMXB stage. Millisecond pulsars with low $\Omega \dot{\Omega}$ may nevertheless have rather high surface temperatures $\left(T_{\mathrm{s}}^{\infty} \gtrsim 10^{5} \mathrm{~K}\right)$.

As in the case of normal NSs, we checked the sensitivity of our results to the choice of $\dot{M}$ and $q$. We found that, while variation of these parameters has a certain effect on the stellar temperature at the LMXB stage, the built-up imbalances, and hence the NS temperature at the MSP stage, are quite insensitive to the choice of $\dot{M}$ and $q$. Note that Fig. 9 implies that when we consider superconducting NSs, $\eta_{1}^{\infty} \gg T^{\infty}$ both at the MSP and LMXB stages (i.e., we are in the so-called 'suprathermal' regime). In this regime, the relaxation rate of the imbalances, determined by $\Delta \Gamma_{1}$, does not depend on temperature, but strongly depends on the imbalance values, $\Delta \Gamma_{1} \propto \eta_{1}^{7}$ (Yakovlev et al. 2001). As a result, although $T^{\infty}$ is sensitive to $\dot{M}$ and $q$ values, the imbalances and NS temperature at the MSP stage are not affected by these parameters.

\section{DISCUSSION}

In this paper we worked under a number of simplifying assumptions. Here we discuss how they may affect our conclusions.

First, we considered the NS model with forbidden DUrca processes. However, for many admissible EOSs these processes may operate in the inner cores of massive NSs. If open, DUrca processes dramatically accelerate particle mutual transformations and hence relaxation of the imbalances. As long as the magnetic field in NS cores does not vanish, the unsuppressed DUrca reactions proceed in flux tubes/normal domains. As a result, the steady-states reached in NSs with open DUrca correspond to much lower values of the imbalances comparing to NSs with closed DUrca, so that the chemical heating in the former stars is not significant. Note also that the central regions in high-mass NSs may be nonsuperconducting (see, e.g., Fig. 1). In these regions particle mutual transformations are not suppressed by proton superconductivity, that also leads to a more rapid relaxation of the imbalances. All this suggests that chemical heating in massive NSs may be inefficient.

Further, in this work we did not account for the nonequilibrium lepton decay process (Alford \& Good 2010):

$$
\mathrm{e}+\mathrm{l} \rightarrow \mu+\mathrm{l}+\nu_{\mathrm{e}}+\bar{\nu}_{\mu}, \quad \mu+\mathrm{l} \rightarrow \mathrm{e}+\mathrm{l}+\nu_{\mu}+\bar{\nu}_{\mathrm{e}} .
$$

Generally, this process is much weaker than MUrca processes (as follows from figure 4 of Alford \& Good 2010, by about eight orders of magnitude). However, when superfluidity/superconductivity strongly suppresses MUrca reactions, lepton decay becomes the main process of particle mutual transformations. Note that Alford \& Good (2010) calculated the non-equilibrium reaction rates for (15) in subthermal regime, when $\mu_{\mathrm{e}}-\mu_{\mu} \ll T$. In our problem we are interested in the opposite (suprathermal) regime, when $\mu_{\mathrm{e}}-\mu_{\mu} \gg T$. Unfortunately, lepton decay in this limit has not been discussed in the literature to our best knowledge, so we did not include this process in this work. However, we believe that lepton decay cannot qualitatively affect our results since it relaxes $\mu_{\mathrm{e}}-\mu_{\mu}$ only, tending to equalize $\eta_{\mathrm{e}}$ and $\eta_{\mu}$, but it is unable to vanish $\eta_{\mathrm{l}}$. Note that, in all our calculations $\eta_{\mathrm{e}}$ and $\eta_{\mu}$ are rather close to each other, which means that even if 

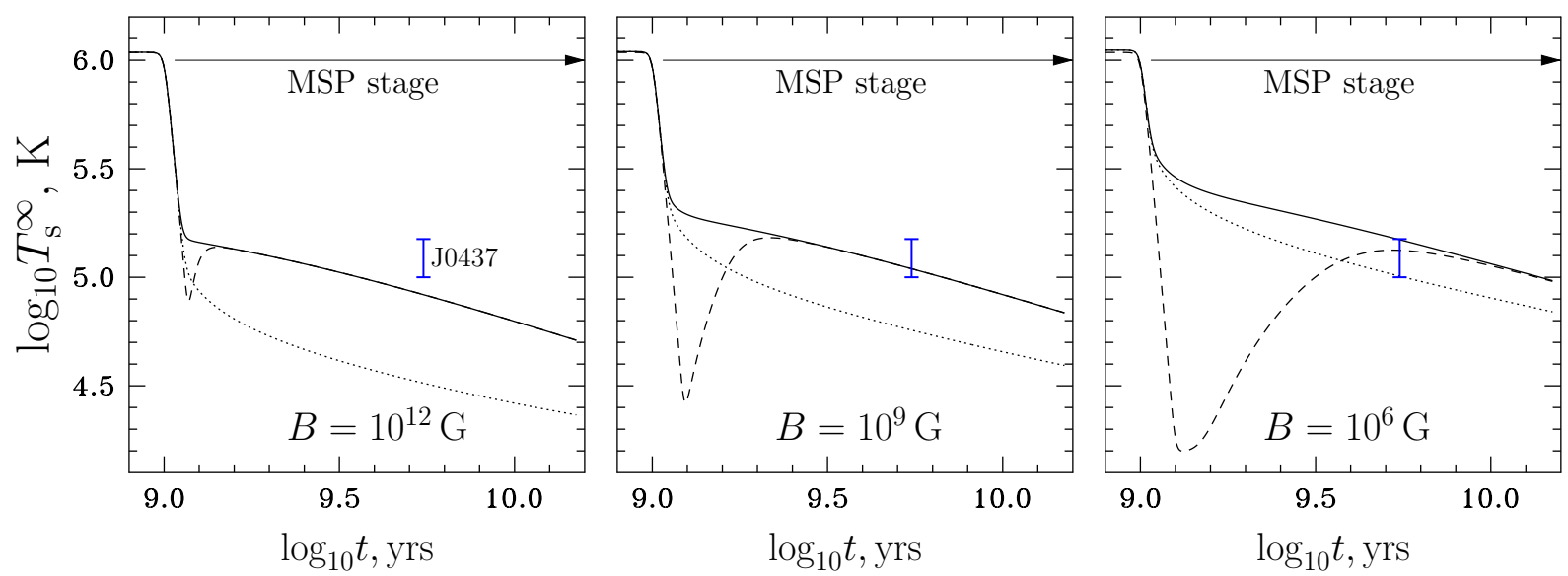

Figure 8. $T_{\mathrm{S}}^{\infty}$ normalized to $10^{5} \mathrm{~K}$ versus time. Protons are superconducting in the whole NS core. Dotted lines are plotted assuming that there is no spin-down $(\dot{\Omega}=0)$, dashed lines - no compression due to accretion at the LMXB stage, solid lines account for both compression due to accretion and NS spin-down. Three panels correspond to three values of the magnetic field (from left to right): $B=10^{12}, 10^{9}$, and $10^{6} \mathrm{G}$. Error bars show $T_{\mathrm{s}}^{\infty}$ for the pulsar J0437.
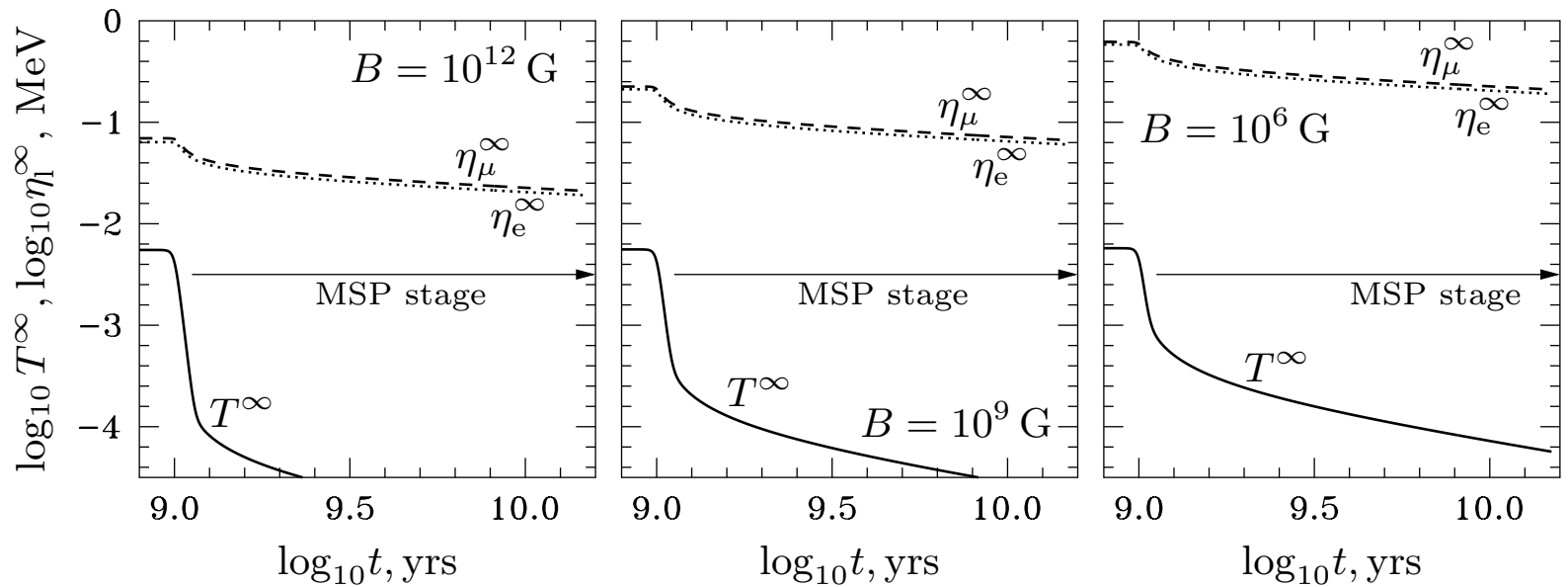

Figure 9. The same as in Fig. 6, but protons are superconducting. It is assumed that $\dot{\Omega}=0$. Three panels correspond to three values of the magnetic field in the core.

the lepton decay was efficient, it would equalize $\eta_{\mathrm{e}}$ and $\eta_{\mu}$ at some average value, but would not significantly affect the evolution of stellar temperature.

Next, for simplicity we assumed that neutrons in the star are nonsuperfluid (normal). Neutron superfluidity may affect the chemical heating in two ways. First, it introduces the Cooper pairing neutrino emission process, a strong cooling agent in NSs with internal temperatures comparable, but smaller than the neutron critical temperature (Yakovlev et al. 2001). Note that, due to rather low temperatures of MSPs, the Cooper pairing neutrino emission process is negligible in MSPs. This process may, however, increase the NS cooling rate at the LMXB stage, when an NS is noticeably hotter. As a result, the equilibrium temperature at the LMXB stage may appear to be a bit smaller than in our simulations.

The second effect of neutron superfluidity concerns the suppression of particle mutual transformations in MUrca re- actions. ${ }^{5}$ It directly reduces the cooling/heating rate (depending on the ratio of $\eta_{1} / T$; see Fernández \& Reisenegger 2005) of an NS due to MUrca processes and, in addition, reduces the relaxation rates of the imbalances, allowing $\eta_{\mathrm{e}}$ and $\eta_{\mu}$ to reach higher values (Yanagi, Nagata, \& Hamaguchi 2020). However, such a suppression of MUrca reactions is only efficient as long as $\eta_{1} \lesssim \Delta_{n}$, where $\Delta_{n}$ is the neutron superfluid energy gap. Once $\eta_{1}$ have reached $\Delta_{\mathrm{n}}$, the subsequent growth of the imbalances leads to less and less efficient suppression of the reaction rates by neutron superfluidity. In other words, neutron superfluidity has a significant impact on the MUrca reaction rates only if $\eta_{1} \lesssim \Delta_{\text {n }}$ (or $\eta_{1}^{\infty} \lesssim \Delta_{\mathrm{n}}^{\infty}$ ) in a substantial fraction of the NS core. However, observations of cooling NSs and NSs in LMXBs seem to indicate (e.g., Gusakov et al. 2004; Page et al. 2004; Gusakov et al. 2005; Shternin et al. 2011; Page et al. 2011;

\footnotetext{
5 Bremsstrahlung processes with neutrons are also suppressed by neutron superfluidity.
} 
Elshamouty et al. 2013; Ho et al. 2015; Beloin et al. 2018; Kantor et al. 2020, 2021; Shternin et al. 2021) that the maximum value of the redshifted neutron energy gap in the core should not exceed $\Delta_{\mathrm{n}}^{\infty} \lesssim 0.07 \mathrm{MeV}$, and is even lower away from the maximum. This constraint also does not contradict microscopic calculations (e.g., Lombardo \& Schulze 2001; Yakovlev et al. 1999; Gezerlis et al. 2014; Dong et al. 2014; Ding et al. 2016; Sedrakian \& Clark 2019). At the same time, our results imply that the chemical heating is capable of maintaining the surface temperature $T_{\mathrm{s}}^{\infty} \approx 10^{5} \mathrm{~K}$ for the parameters of PSR J0437-4715 as an example, only if the imbalances are rather large, $\eta_{1}^{\infty}>0.1 \mathrm{MeV}$, i.e., $\eta_{1}^{\infty}>\Delta_{\mathrm{n}}^{\infty}$. This suggests that neutron superfluidity should not affect our results qualitatively, although some quantitative effect is of course expected.

Now, let us comment on our model of proton superconductivity. When considering superconducting NSs, we assumed that proton superconductivity extends over the whole NS core. Only in this case our conclusions about the thermal states of superconducting MSPs are valid. If $\Delta_{\mathrm{p}} \lesssim T$ in some part of the core, the imbalances effectively relax through unsuppressed MUrca reactions there, and no effective chemical heating is possible (see González-Jiménez et al. 2015). Moreover, to find the thermal state of a superconducting spinning down MSP with $B=0$, we assumed that the redshifted proton critical temperature is constant throughout the core. ${ }^{6}$ In contrast, González-Jiménez et al. (2015) considered a more realistic bell-shaped profile of $T_{\mathrm{cp}}$. They found that the redshifted imbalances first grow up to the lowest value of $\Delta_{\mathrm{p}}^{\infty}$ in the core, then nonequilibrium MUrca reactions switch on in the volume fraction in which $\eta_{1}^{\infty}>\Delta_{\mathrm{p}}^{\infty}$ and prevent subsequent growth of the imbalances in the NS core. This means that our results for the flat profile with $T_{\mathrm{cp}}^{\infty}=2 \times 10^{9} \mathrm{~K}$ are equally valid for any bell-shaped profile with the minimum value of $T_{\mathrm{cp}}^{\infty}$ equal to $2 \times 10^{9} \mathrm{~K}$. Note that microscopic calculations (see, e.g., Ding et al. 2016 and Fig. 1) imply that in our stellar model the chosen value of $T_{\mathrm{cp}}^{\infty}=2 \times 10^{9} \mathrm{~K}$ can be considered as a lower limit on the real minimum of $T_{\mathrm{cp}}^{\infty}$. Thus, the derived surface temperature (see the last panel in Fig. 4) is the estimate from below for the real one.

Finally, modeling of the Roche-lobe decoupling phase predicts (Tauris 2012) that in the end of the LMXB phase an NS experiences a spin-down torque, which can substantially reduce the NS spin frequency. Such a spin-down would additionally compress the NS matter. However, as we checked, this effect does not significantly affect our results, since the matter compression in the course of accretion is much more efficient.

6 We have to specify the $T_{\mathrm{cp}}^{\infty}$-profile to calculate the reduction factors for MUrca processes by proton superconductivity. In the case of non-vanishing magnetic field, $B \neq 0$, we do not need to calculate these factors, because we treat MUrca reactions outside the flux tubes/normal domains as fully suppressed in this case (i.e., we neglect a contribution of MUrca reactions outside the flux tubes/normal domains in comparison to that inside the flux tubes/normal domains). This approach is justified for typical LMXB/MSP temperatures and magnetic fields $B \gtrsim 10^{6} \mathrm{G}$ as long as $\eta_{1}^{\infty}<\Delta_{\mathrm{p}}^{\infty}$ in the whole stellar core. The latter condition is fulfilled for most of our numerical examples for the critical temperature profiles with $T_{\mathrm{cp}}^{\infty} \gtrsim 2 \times 10^{9} \mathrm{~K}$, see footnote 4 for details.

\section{CONCLUSIONS}

This work studies two aspects of the chemical heating of MSPs. First, we analyze the effect of stellar core magnetic field on the heating of superconducting MSPs. Second, we look into the role of the preceding accretion at the LMXB stage on building up the chemical potential imbalances and their effect on the subsequent thermal evolution of MSP.

In the previous studies of Petrovich \& Reisenegger (2010) and González-Jiménez et al. (2015) it was found that an MSP should be effectively heated by the rotochemical mechanism if proton and/or neutron energy gaps are sufficiently large in the whole stellar core. This is required for an efficient heating since the redshifted chemical potential imbalances $\eta_{1}^{\infty}$ tend to grow to the lowest value of the corresponding redshifted gap in the course of NS compression. Higher values of the gaps lead to larger imbalances, when an NS reaches the steady state. Since the heating rate due to the non-equilibrium MUrca processes is proportional to $\eta_{1}^{\infty}$ in this state, larger gaps result in hotter NSs in the steady state. Adopting the flat critical temperature profile throughout the core, $T_{\mathrm{cp}}^{\infty}=$ const, we confirmed that in the absence of the magnetic field the imbalances tend to grow to the value of the proton superfluid gap $\left(\eta_{1}^{\infty} \approx \Delta_{\mathrm{p}}^{\infty}\right)$. However, we found that even small magnetic field in the core (in our numerical examples, $B \gtrsim 10^{6} \mathrm{G}$ ) may terminate this growth at some value of $\eta_{1}^{\infty}<\Delta_{\mathrm{p}}^{\infty}$. This happens because the core magnetic field destroys superconductivity in some volume fraction proportional to the field value, so that unsuppressed nonequilibrium reactions may proceed there and relax the imbalances. As a result, the steady state corresponds to lower $\eta_{1}^{\infty}$ and lower surface temperatures at higher $B$. Thus, the core magnetic field reduces the efficiency of the rotochemical heating even if proton superconductivity extends over the whole NS core. In particular, sufficiently high values of the core magnetic field (such as $B=10^{12} \mathrm{G}$ ) significantly complicate explanation of the surface temperature of J0437 within the rotochemical heating scenario.

On the contrary, the preceding accretion at the LMXB stage may help to bring the imbalances to higher values even at non-zero magnetic field in the core. It appears to be possible because of the strong compression of NS interiors caused by the accreted matter. As a result, after accretion ceases, nonequilibrium processes heat an MSP, and the latter may stay warm $\left(T_{\mathrm{s}}^{\infty} \gtrsim 10^{5} \mathrm{~K}\right)$ for about billion years even in the absence of any spin-down (see Fig. 8). In this respect it is worth noting that all the known effective reheating mechanisms operate by transforming the rotational energy to the thermal energy (Alpar et al. 1984; Reisenegger 1995; Gusakov et al. 2015). Thus, currently, it is believed that the higher is the loss of the rotational energy by an MSP, the larger is its surface temperature. Here we find that it is not necessary the case, since preceding accretion may 'charge' the MSP core with the chemical energy, thus providing the long-lasting energy source for the MSP's subsequent thermal luminosity. This means that MSPs with low spin-down rates may still be warm.

Finally, it is worth noting that the magnetic field in superconducting cores of ordinary pulsars may also reduce the efficiency of rotochemical heating in these objects. We leave a detailed discussion of the related effects for a future work. 


\section{ACKNOWLEDGMENTS}

We thank Andreas Reisenegger for valuable comments on the draft version of the manuscript. EK was supported by the Russian Science Foundation (grant number 19-1200133).

\section{DATA AVAILABILITY}

The data underlying this article are available in the article.

\section{REFERENCES}

Alford M. G., Good G., 2010, Phys. Rev. C, 82, 055805

Alpar M. A., Pines D., Anderson P. W., Shaham J., 1984, ApJ, 276,325

Antoniadis J., Tauris T. M., Ozel F., Barr E., Champion D. J., Freire P. C. C., 2016, arXiv e-prints, arXiv:1605.01665

Baiko D. A., Yakovlev D. G., 1999, A\&A, 342, 192

Beloin S., Han S., Steiner A. W., Page D., 2018, Phys. Rev. C, 97, 015804

Bhattacharya S., Heinke C. O., Chugunov A. I., Freire P. C. C., Ridolfi A. r., Bogdanov S., 2017, MNRAS, 472, 3706

Bogdanov S. et al., 2019, ApJ, 887, L25

Boztepe T., Göğüs E., Güver T., Schwenzer K., 2019, arXiv eprints, arXiv:1910.04448

Cruces M., Reisenegger A., Tauris T. M., 2019, MNRAS, 490, 2013

de Gennes P. G., 1967, Nuclear Phys. A, 90, 706

Ding D., Rios A., Dussan H., Dickhoff W. H., Witte S. J., Carbone A., Polls A., 2016, Phys. Rev. C, 94, 025802

Ding D., Rios A., Dussan H., Dickhoff W. H., Witte S. J., Carbone A., Polls A., 2016, Phys. Rev. C, 94, 025802

Dommes V. A., Gusakov M. E., 2017, MNRAS, 467, L115

Dommes V. A., Gusakov M. E., Shternin P. S., 2020, Phys. Rev. D, 101, 103020

Dong J. M., Lombardo U., Zuo W., 2014, Physics of Atomic Nuclei, 77,1057

Durant M., Kargaltsev O., Pavlov G. G., Kowalski P. M., Posselt B., van Kerkwijk M. H., Kaplan D. L., 2012, ApJ, 746, 6

Elshamouty K. G., Heinke C. O., Sivakoff G. R., Ho W. C. G., Shternin P. S., Yakovlev D. G., Patnaude D. J., David L., 2013, ApJ, 777, 22

Fernández R., Reisenegger A., 2005, ApJ, 625, 291

Gamow G., Schoenberg M., 1941, Physical Review, 59, 539

Gezerlis A., Pethick C. J., Schwenk A., 2014, ArXiv e-prints

Ghosh P., Lamb F. K., 1979, ApJ, 234, 296

Glampedakis K., Andersson N., Samuelsson L., 2011, MNRAS, 410,805

Gonzalez D., Reisenegger A., 2010, A\&A, 522, A16

González-Caniulef D., Guillot S., Reisenegger A., 2019, MNRAS, 490,5848

González-Jiménez N., Petrovich C., Reisenegger A., 2015, MNRAS, 447, 2073

Goriely S., Chamel N., Pearson J. M., 2013, Phys. Rev. C, 88, 024308

Gusakov M. E., 2019, MNRAS, 485, 4936

Gusakov M. E., Chugunov A. I., 2020, Phys. Rev. Lett., 124, 191101

Gusakov M. E., Chugunov A. I., 2021, Phys. Rev. D, 103, L101301

Gusakov M. E., Kaminker A. D., Yakovlev D. G., Gnedin O. Y., 2004, A\&A, 423, 1063

Gusakov M. E., Kaminker A. D., Yakovlev D. G., Gnedin O. Y., 2005, MNRAS, 363, 555

Gusakov M. E., Kantor E. M., Reisenegger A., 2015, MNRAS, 453, L36
Hartle J. B., 1967, ApJ, 150, 1005

Hartle J. B., Thorne K. S., 1968, ApJ, 153, 807

Haskell B., Sedrakian A., 2018, Superfluidity and Superconductivity in Neutron Stars, Rezzolla L., Pizzochero P., Jones D. I., Rea N., Vidaña I., eds., Vol. 457, p. 401

Ho W. C. G., Elshamouty K. G., Heinke C. O., Potekhin A. Y., 2015, Phys. Rev. C, 91, 015806

Ho W. C. G., Heinke C. O., Chugunov A. I., 2019, ApJ, 882, 128

Kantor E. M., Gusakov M. E., Dommes V. A., 2020, Phys. Rev. Lett., 125, 151101

Kantor E. M., Gusakov M. E., Dommes V. A., 2021, Phys. Rev. D, 103, 023013

Kargaltsev O., Pavlov G. G., Romani R. W., 2004, ApJ, 602, 327

Lifshitz E. M., Pitaevskii L. P., 1980, Statistical Physics, Part 2. Pergamon Press, Oxford

Lombardo U., Schulze H.-J., 2001, in Lecture Notes in Physics, Berlin Springer Verlag, Vol. 578, Physics of Neutron Star Interiors, Blaschke D., Glendenning N. K., Sedrakian A., eds., p. 30

Özel F., Psaltis D., Narayan R., Santos Villarreal A., 2012, ApJ, 757,55

Page D., Lattimer J. M., Prakash M., Steiner A. W., 2004, ApJS, 155,623

Page D., Prakash M., Lattimer J. M., Steiner A. W., 2011, Phys. Rev. Lett., 106, 081101

Papitto A., Menna M. T., Burderi L., di Salvo T., Riggio A., 2008, MNRAS, 383, 411

Papitto A., Riggio A., Burderi L., di Salvo T., D'Aí A., Iaria R., 2011, A\&A, 528, A55

Patruno A., 2010, ApJ, 722, 909

Pearson J. M., Chamel N., Potekhin A. Y., Fantina A. F., Ducoin C., Dutta A. K., Goriely S., 2018, MNRAS, 481, 2994

Pearson J. M., Chamel N., Potekhin A. Y., Fantina A. F., Ducoin C., Dutta A. K., Goriely S., 2019, MNRAS, 486, 768

Petrovich C., Reisenegger A., 2010, A\&A, 521, A77

Potekhin A. Y., Chabrier G., Yakovlev D. G., 1997, A\&A, 323, 415

Potekhin A. Y., Fantina A. F., Chamel N., Pearson J. M., Goriely S., 2013, A\&A, 560, A48

Pringle J. E., Rees M. J., 1972, A\&A, 21, 1

Rangelov B., Pavlov G. G., Kargaltsev O., Reisenegger A., Guillot S., van Kerkwijk M. H., Reyes C., 2017, ApJ, 835, 264

Reardon D. J. et al., 2016, MNRAS, 455, 1751

Reisenegger A., 1995, ApJ, 442, 749

Reisenegger A., 1997, ApJ, 485, 313

Reisenegger A., Jofré P., Fernández R., Kantor E., 2006, ApJ, 653,568

Schaab C., Weber F., Weigel M. K., 1998, A\&A, 335, 596

Schwenzer K., Boztepe T., Güver T., Vurgun E., 2017, MNRAS, 466, 2560

Sedrakian A., Clark J. W., 2019, European Physical Journal A, 55,167

Shternin P. S., Baldo M., Haensel P., 2018, Physics Letters B, 786, 28

Shternin P. S., Ofengeim D. D., Ho W. C. G., Heinke C. O., Wijngaarden M. J. P., Patnaude D. J., 2021, MNRAS, 506, 709

Shternin P. S., Yakovlev D. G., Heinke C. O., Ho W. C. G., Patnaude D. J., 2011, MNRAS, 412, L108

Tauris T. M., 2012, Science, 335, 561

Villain L., Haensel P., 2005, A\&A, 444, 539

Wood T. S., Graber V., Newton W. G., 2020, arXiv e-prints, arXiv:2011.02873

Yakovlev D. G., Kaminker A. D., Gnedin O. Y., Haensel P., 2001, Phys. Rep., 354, 1

Yakovlev D. G., Levenfish K. P., Shibanov Y. A., 1999, Sov. Phys.- Usp., 42, 737

Yanagi K., Nagata N., Hamaguchi K., 2020, MNRAS, 492, 5508 